\title{
Recidiva de linfangiomas quísticos retroperitoneales con dificultad para la exéresis quirúrgica. ¿Existen otras alternativas terapéuticas?
}

\section{Recurrent of retroperitoneal cystic lymphangiomas with difficulty for surgical resection. Is there other therapeutic options?}

\section{Sr. Director:}

El linfangioma es una malformación congénita benigna caracterizada por la dilatación de los vasos linfáticos, siendo inusual la localización intraabdominal. La elevada morbilidad y el porcentaje de recidiva hacen que investiguemos sobre otras modalidades terapéuticas para situaciones irresecables o que supongan una morbilidad importante.

Presentamos el caso de un paciente varón de 16 años, sin antecedentes personales, que a consecuencia de un síndrome miccional de un mes y medio de evolución es diagnosticado de una masa retroperitoneal izquierda que comprimía y desplazaba los uréteres y la vejiga, ocupando la pelvis y ascendiendo hasta el hilio renal (fig. 1A). Se realizó exéresis radical, siendo el resultado anatomopatológico de linfangioma quístico. Se reintervino tras evidenciar una recidiva a los dos años de la cirugía, adyacente al músculo psoas hasta el músculo psoas ilíaco (fig. 1B). Se constató una nueva recidiva a nivel pélvico izquierdo, adyacente al hueso ilíaco, y por detrás del músculo psoas ilíaco, que se procedió a resecar. La histología de las piezas resecadas en las diferentes intervenciones quirúrgicas no constató evidencia de enfermedad residual. Actualmente, el paciente está asintomático y libre de enfermedad.

También presentamos el caso de una paciente mujer de 21 años, sin antecedentes personales, que ingresó en nuestro servicio por dolor abdominal de un mes de evolución. Fue intervenida quirúrgicamente en otro centro por una masa intraabdominal retroperitoneal, existiendo enfermedad residual tras el estudio histológico. No se realizaron controles evolutivos posteriores, siendo remitida a nuestro servicio por clínica similar a la previa. Se evidenció la existencia de neoplasia a nivel retroperitoneal, con infiltración de la vía biliar extrahepática y la cabeza pancreática, que englobaba la arteria hepática y la vena porta (fig. 1C). Se realizó exéresis quirúrgica con informe anatomopatológico de linfangioma quístico, sin enfermedad residual (fig. 1D). El postoperatorio fue infausto por hemoperitoneo secundario a necrosis portal, con desarrollo de fallo multiorgánico y fallecimiento de la paciente.
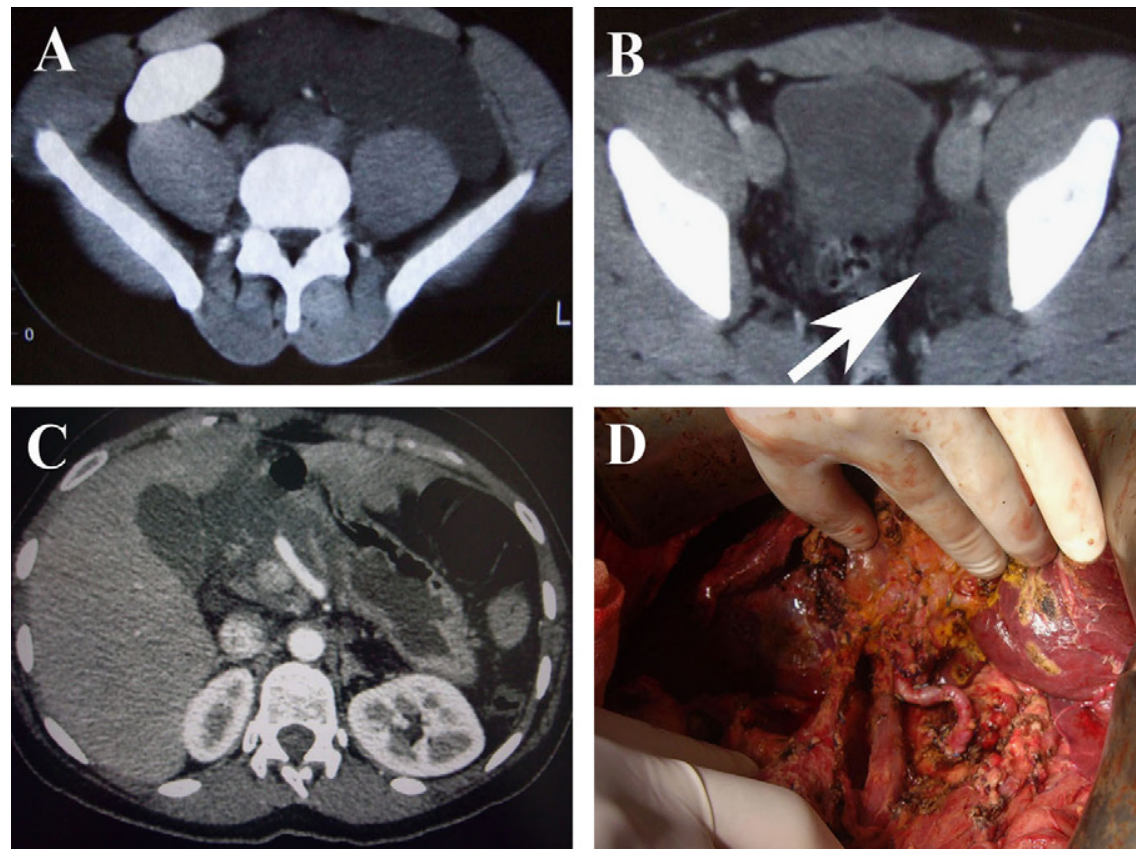

Figura 1 - A) Imagen de TC que muestra la lesión a nivel de la pelvis. B) Imagen de TC que muestra la recidiva adyacente al músculo psoas-iliaco. C) Imagen de TC que muestra la lesión a nivel del hilio hepático. D) Lecho quirúrgico tras la exéresis de la recidiva del linfangioma quístico. 
El linfangioma es una malformación congénita benigna del sistema linfático, con una incidencia de 1:100.000 nacimientos $^{1}$. Se caracteriza por ser una patología benigna formada por la dilatación de los vasos linfáticos, generalmente a nivel cervical, axilar o mediastínico, siendo inusual la localización intraabdominal ${ }^{2}$, que representa del $5-20 \%$ de los casos $^{1}$. La lesión se sitúa en el mesenterio en un 93\% de los casos, habiéndose descrito en el retroperitoneo en un $7 \%^{3}$. Sólo Ozdemir et $\mathrm{al}^{4}$ y Canataroglu et $\mathrm{al}^{5}$ han descrito una localización a nivel del hilio hepático.

Aunque su etiología no está clara, se acepta su origen congénito, siendo otras posibles causas: traumatismo abdominal, neoplasia, degeneración ganglionar y obstrucción linfática ${ }^{6}$. Los linfangiomas se clasifican en capilares, cavernosos y quísticos, habiéndose localizado de forma intraabdominal los dos últimos ${ }^{7}$.

La presentación clínica es inespecífica, cursando de forma asintomática, o bien puede presentarse como masa abdominal, pseudoascitis, abdomen agudo, distensión abdominal, episodios de suboclusión y dolor abdominal crónico ${ }^{1-8}$; entre otras complicaciones, se han descrito la hemorragia y la infección intralesional ${ }^{8}$.

El diagnóstico se basa en la ecografía, que muestra una lesión circunscrita, anecoica y septada en las lesiones multiloculadas ${ }^{1}$. Tanto la tomografía computarizada como la resonancia magnética proporcionan información acerca del tamaño, la extensión y la relación con estructuras vecinas ${ }^{8}$. Se debe realizar el diagnóstico diferencial con otras patologías malignas retroperitoneales, como tumores quísticos pancreáticos, metástasis ganglionares retroperitoneales, sarcomas retroperitoneales, lipomas, hematomas, linfoceles o abscesos ${ }^{7,8}$.

El tratamiento de elección en pacientes sintomáticos es la resección neoplásica ${ }^{1-8}$. Las principales complicaciones que pueden presentarse son la recidiva (15-53\%), por la imposibilidad de llevar a cabo una resección completa, y la morbilidad secundaria a lesión de estructuras adyacentes; la recidiva es infrecuente con márgenes de resección libre de enfermedad tras resección neoplásica ${ }^{9}$. Para evitar las posibles complicaciones de la cirugía y la alta morbilidad perioperatoria, se ha recurrido a tratamiento mediante agentes esclerosantes intralesionales como el Picibanil ${ }^{\mathbb{R}}$ (OK-432), principalmente aplicado en edades tempranas, en lesiones de la cabeza y el cuello, con tasas de curación de hasta el 87\%. El mecanismo de acción de este agente es la inducción de una reacción inflamatoria, con migración de neutrófilos y monocitos hacia los espacios quísticos, donde se ve incrementado el nivel de mediadores de la inflamación, como interferón- $\gamma$, TNF, IL-1, IL-2 e IL-6, entre otras ${ }^{10}$.

Otras modalidades alternativas a la cirugía: a) radioterapia; b) radioquimioterapia; c) quimioterapia; d) láser de $\mathrm{CO}_{2}$ y Nd-Yag; e) coagulador de argón; f) tratamientos esclerosantes, como el OK-432, la bleomicina, las soluciones alcohólicas, el ácido acético y la doxiciclina ${ }^{9,10}$. El problema que se presenta con los agentes esclerosantes es la extravasación de estas sustancias fuera de la lesión ${ }^{9}$. Esta modalidad terapéutica se presenta como alternativa paliativa en situaciones en las que no es posible la exéresis quirúrgica, habiéndose obtenido buenos resultados en pacientes adultos con localización de la lesión a nivel intraabdominal ${ }^{9}$. Se han recogido diez casos en la literatura médica en los que se ha realizado escleroterapia en linfangiomas de diferente localización intraabdominal, empleándose OK-432, ácido acético, bleomicina y doxiciclina.

Estudios retrospectivos con mayor muestra serán necesarios para conocer el verdadero valor terapéutico.

\section{B I B L I O G R A F Í A}

1. De Lagausie P, Bonnard A, Berrebi D, Lepretre O, Statopoulos L, Delarue A, et al. Abdominal lymphangiomas in children: Interest of the laparoscopic approach. Surg Endosc. 2007;21:1153-7.

2. Pereira Gallardo S, Gómez Torres FJ, Torres Olivera FJ. Cystic lymphangioma of the adrenal glands. Case report. Arch Esp Urol. 2007;60:187-9.

3. Güvenç BH, Ekingen G, Tuzlaci A, Senel U. Diffuse neonatal abdominal lymphangiomatosis: Management by limited surgical excision and sclerotherapy. Pediatr Surg Int. 2005;21: 595-8.

4. Ozdemir H, Kocakoc E, Bozgeyik Z, Cobanoglu B. Recurrent retroperitoneal cystic lymphangioma. Yonsei Med J. $2005 ; 46: 715-8$.

5. Canataroglu A, Gumurdulu Y, Gumurdulu D, Balal M, Tuncer I, Sagliker Y. Coexistence of cystic intra-abdominal lymphangiomas and diffuse venous haemangiomas in adult life. Neth J Med. 2003;61:95-7.

6. Mohite PN, Bhatnagar AM, Parikh SN. A huge omental lymphangioma with extension into labia majorae: A case report. BMC Surg. 2006;27:18.

7. Erdem S, Iskender C, Avsar AF, Altundag OB, Ustunyurt E. Benign cystic lymphangioma presenting as a pelvic mass. J Obstet Gynaecol Res. 2006;32:628-30.

8. Arzoz M, Ibarz LL, Areal J, González C, Ruiz J, Saladié JM. Cystic lymphangioma. Our experience. Actas Urol Esp. 2006;30: 723-7.

9. Luzzatto C, Midrio P, Tchaprassian Z, Guglielmi M. Sclerosing treatment of lymphangiomas with OK-432. Arch Dis Child. 2000;82:316-8.

10. Knipping S, Goetze G, Neumann K, Bloching M. Sclerotherapy of cervical cysts with Picibanil (OK-432). Eur Arch Otorhinolaryngol. 2007;264:423-7.

\section{P. Menéndez*, D. Padilla, P. Villarejo y J. Martín}

Servicio de Cirugía General y de Aparato Digestivo, Hospital General de Ciudad Real, Ciudad Real, España

*Autor para correspondencia.

Correos electrónicos: pablomensan@hotmail.com, pablo.menendez.sanchez@gmail.com (P. Menéndez). 